\title{
GA-A23849
}

\section{SUSTAINED STABILIZATION OF THE RESISTIVE WALL MODE BY PLASMA ROTATION IN THE DIII-D TOKAMAK}

by

A.M. GAROFALO, E.J. STRAIT, L.C. JOHNSON, R.J. LA HAYE, E.A. LAZARUS, G.A. NAVRATIL, M. OKABAYASHI, J.T. SCOVILLE, T.S. TAYLOR, A.D. TURNBULL, and the DIII-D TEAM 


\section{DISCLAIMER}

This report was prepared as an account of work sponsored by an agency of the United States Government. Neither the United States Government nor any agency thereof, nor any of their employees, makes any warranty, express or implied, or assumes any legal liability or responsibility for the accuracy, completeness, or usefulness of any information, apparatus, product, or process disclosed, or represents that its use would not infringe privately owned rights. Reference herein to any specific commercial product, process, or service by trade name, trademark, manufacturer, or otherwise, does not necessarily constitute or imply its endorsement, recommendation, or favoring by the United States Government or any agency thereof. The views and opinions of authors expressed herein do not necessarily state or reflect those of the United States Government or any agency thereof. 


\title{
SUSTAINED STABILIZATION OF THE RESISTIVE WALL MODE BY PLASMA ROTATION IN THE DIII-D TOKAMAK
}

\author{
by \\ A.M. GAROFALO, ${ }^{*}$ E.J. STRAIT, L.C. JOHNSON, ${ }^{\dagger}$ R.J. LA HAYE, \\ E.A. LAZARUS, ${ }^{\ddagger}$ G.A. NAVRATIL, ${ }^{*} M$. OKABAYASHI, ${ }^{\dagger}$ \\ J.T. SCOVILLE, T.S. TAYLOR, A.D. TURNBULL, and the DIII-D TEAM
}

This is a preprint of a paper to be submitted for publication in Phys. Rev. Lett.

*Columbia University, New York, New York.

${ }^{\dagger}$ Princeton Plasma Physics Laboratory, Princeton, New Jersey.

$\ddagger_{\text {Oak Ridge National Laboratory, Oak Ridge, Tennessee. }}$

\author{
Work supported by \\ the U.S. Department of Energy under \\ Contract Nos. DE-AC03-99ER54463, DE-FG02-89ER53297, \\ DE-AC05-00OR22725, and DE-AC02-76CH03073
}

GENERAL ATOMICS PROJECT 30033 OCTOBER 2001 


\section{ABSTRACT}

A path to sustained stable operation, at plasma pressure up to twice the ideal magnetohydrodynamic (MHD) $n=1$ free-boundary pressure limit, has been discovered in the DIII-D tokamak. Tuning the correction of the intrinsic magnetic field asymmetries so as to minimize plasma rotation decay during the high beta phase and increasing the angular momentum injection, have allowed maintaining the plasma rotation above that needed for stabilization of the resistive wall mode (RWM). A new method to determine the improved magnetic field correction uses feedback to sense and minimize the resonant plasma response to the non-axisymmetric field. At twice the free-boundary pressure limit, a disruption precursor is observed, which is consistent with having reached the "ideal wall" pressure limit predicted by stability calculations. 
The long time-scale stabilization of a hot plasma by a resistive wall is an important test of magnetohydrodynamic (MHD) theory. It is also a problem of great practical significance for controlled nuclear fusion research. Standard ideal MHD theory predicts that long wavelength "kink" modes will limit the achievable pressure in tokamaks and other toroidal magnetic confinement devices. The semi-empirical "Troyon scaling" [1] expresses the kink mode stability limit in terms of a "normalized beta," $\beta_{\mathrm{N}}=\beta /\left(I_{\mathrm{p}} / a B\right)$, where $I_{\mathrm{p}}$ is the toroidal plasma current, $a$ is the plasma minor radius, $B$ is the magnetic field strength, and $\beta=\langle p\rangle /\left(B^{2} / 2 \mu_{\mathrm{o}}\right)$ is the dimensionless plasma pressure. The limiting value of $\beta_{\mathrm{N}}$ depends on details of the plasma's shape and internal distribution of pressure and magnetic field. Kink modes can be stabilized by the eddy currents that the instability induces in a nearby perfectly conducting wall, thus raising the maximum $\beta_{\mathrm{N}}$. However, if the wall has finite conductivity, the kink mode is instead converted to a slowly growing resistive wall mode (RWM) [2]. Thus, the RWM occurs in the presence of a resistive wall, under conditions where the ideal kink mode would be unstable without a wall but stable with a perfectly conducting wall. Modifications to the simple ideal MHD theory $[3,4]$ have predicted that sufficient plasma rotation can stabilize the RWM. However, in previous experiments, small amplitude RWMs with toroidal mode number $n=1$ were detected and the plasma rotation decreased whenever $\beta_{\mathrm{N}}$ exceeded the no-wall $\beta_{\mathrm{N}}$ limit, leading to beta rollover or collapse $[5,6]$.

Achieving Sustained High Performance Near Twice the No-Wall Limit. A crucial step in maintaining sufficient rotation at high beta was to minimize toroidal asymmetries in the magnetic field using a set of correction coils [7]. With minimized rotation decay, the next step was to increase the angular momentum injection to maintain $\beta_{\mathrm{N}}$ above the no-wall limit for a longer period of time. The attempt led to discharges like the ones shown in Fig. 1, which demonstrate the first sustained stabilization of the RWM by plasma rotation. A careful increase in the angular momentum input is realized by controlling the neutral beam injected (NBI) power (which also drives the rotation as all beams are co-injected with $I_{p}$ ) through feedback on the stored energy. In the first of the two discharges (106535) the requested stored energy target continuously drives up the value of $\beta_{\mathrm{N}}$, while the plasma current ramp up drives down the plasma internal inductance $\ell_{\mathrm{i}}$ and the no-wall $\beta_{\mathrm{N}}$ limit, which scales as $2.4 \ell_{\mathrm{i}}$ in these plasmas, according to ideal MHD stability calculations. $\beta_{\mathrm{N}}$ reaches double the no-wall limit, at which point the plasma disrupts due to a rapidly growing instability. In the second discharge of Fig. 1 (107603) the requested stored energy target and the current ramp up were moderated just enough to avoid the disruption. In this plasma $\beta_{\mathrm{N}}$ is maintained $\geq 50 \%$ above the $n=1$ nowall limit for up to $\geq 1.5$ seconds, or about 250 times the wall time constant, $\tau_{\mathrm{w}}$ (eddy current decay time for penetration of the $n=1$ mode) and 10 times the energy confinement 
time, $\tau_{E}$. In this discharge, a power supply fault terminates the plasma current ramp up. By then, however, $\tau_{\mathrm{E}}$ is decreasing rapidly, apparently due to the gradual increase of the edge density in these discharges: Higher edge density reduces the penetration of the NBI power, non-linearly degrading the internal transport barrier [8] which was formed with high power NBI in the low density target plasma. Throughout the high beta phase the plasma rotation frequency $f_{\mathrm{p}}=\omega_{\mathrm{p}} / 2 \pi$ at the $q=2$ surface is maintained just above the threshold for stabilization of the RWM $(\sim 6 \mathrm{kHz})$. In relative terms this level of rotation is about $\omega_{\mathrm{p}} \tau_{\mathrm{A}} \sim 0.02\left(\tau_{\mathrm{A}}=q R / V_{\mathrm{A}}\right.$ is the Alfvén time) at the $q=2$ surface, where the unstable $n=1 \mathrm{RWM}$ has peak amplitude. A small decay rate is attributed to the increasing plasma density. Torque balance between the neutral beam deposition, the viscous torque and the residual un-corrected magnetic field asymmetries has been achieved in these plasmas, although at a value of the plasma rotation just sufficient to stabilize the RWM. In terms of fusion performance, discharge 107603 sustains a volume averaged $\beta>3 \%$ for a duration $\sim 10 \tau_{\mathrm{E}}$ : a significantly high value compared to highest sustained values in larger experimental fusion devices [9]. These high fusion performance levels are sustained with a current profile having $\ell_{\mathrm{i}} \sim 0.7$, and relatively flat $q$-profile, with $q_{\min }>$ 1.5 and $q_{95}<4$, which result in a plasma that would be highly unstable without the effect of a surrounding conducting wall.

Determination of the No-Wall Beta Limit. Detailed stability calculations confirm that these discharges are well above the stability limit in the absence of a wall, and that the stability limit is approximately $\beta_{\mathrm{N}}=2.4 \ell_{\mathrm{i}}$. Results of several tests of the time dependence of the $n=1$ no-wall $\beta_{\mathrm{N}}$ limit are shown in Fig. 2. First, equilibrium 


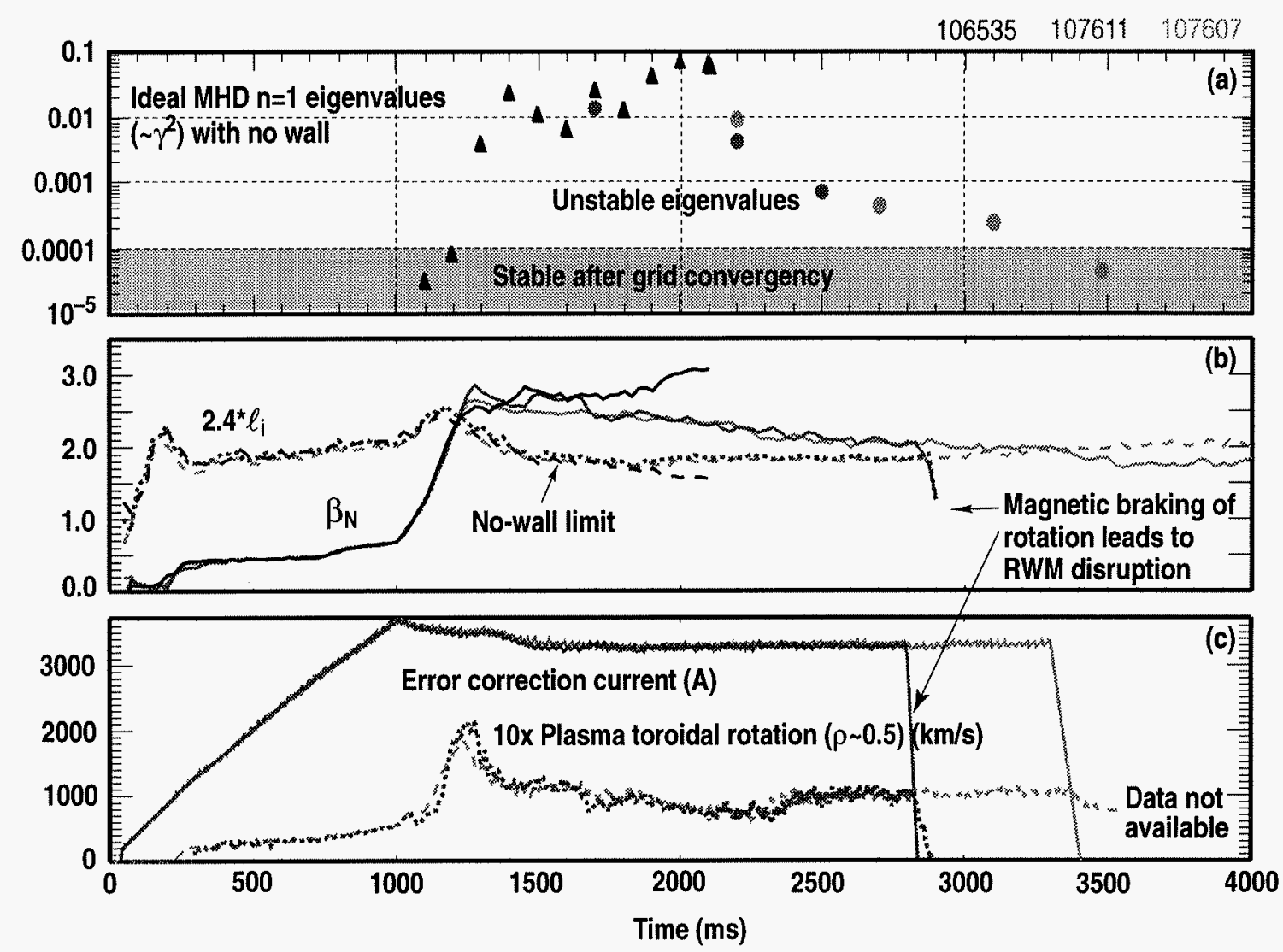

Fig. 2. Numerical and experimental tests of the time evolution of the no-wall $\beta_{N}$ limit. (a) Square of the $n=1$ mode growth rate normalized to the Alfvén frequency calculated with GATO without a wall for three similar discharges (black triangles for 106535, light and dark circles for 107607 and 107611). The predicted growth rate crosses the stability boundary when (b) $\beta_{\mathrm{N}}$ (controlled by feedback) crosses the approximate no-wall $\beta_{\mathrm{N}}$ limit $=2.4 \times \ell_{\mathrm{i}}$, both in the early and late phases of the discharges evolution. (c) Magnetic braking of the plasma rotation, obtained by turning off the magnetic field correction current, leads to beta collapse for discharges with $\beta_{\mathrm{N}}$ exceeding $2.4 \ell_{\mathbf{i}}$ (dark gray), while it leads to just a small rotation drop in discharges with $\beta_{\mathrm{N}}$ just below $2.4 \ell_{\mathrm{i}}$ (light gray).

reconstructions are calculated at several times throughout the high beta phase of discharge 106535 [solid black curve which terminates at $2100 \mathrm{~ms}$ in Fig. 2(b)]. Stability analysis using the GATO ideal MHD stability code [10] shows that in absence of a conducting wall, the $n=1$ kink mode becomes unstable when $\beta_{\mathrm{N}}$ exceeds a value $=2.4 \ell_{\mathrm{i}}$ [triangles in Fig. 2(a)], consistent with previous calculations of the no-wall limit in similar discharges [6]. At this time $(\sim 1200)$ the plasma has just transitioned from low (L) to high $(\mathrm{H})$ confinement mode, and $\ell_{\mathbf{i}}$ is decreasing rapidly. To determine whether the same $\ell_{\mathrm{i}}$ scaling is maintained also at later times, we produced discharges where $\beta_{\mathrm{N}}$ is allowed to decrease slowly below $2.4 \ell_{\mathrm{i}}$ [solid gray curves in Fig. 2(b)], with other plasma parameters similar to the higher beta discharge. The $n=1$ stability calculated at several times shows that the marginal stability boundary is still $2.4 \ell_{\mathrm{i}}$ [dark circles in Fig. 2(a)], continuing even to the termination of the high beta phase. 
We have experimentally confirmed that rotation is critical to the stability of these discharges. Figure 2 also shows the result of an experimental test of the no-wall limit calculation for these two discharges, using magnetic braking of the plasma rotation [11]. In discharge 107607 the correction coil current is turned off when $\beta_{\mathrm{N}}$ has just decreased below the predicted no-wall limit. The increased magnetic field asymmetry causes the plasma rotation to decrease to a level where a new torque equilibrium is established, and the plasma survives at constant beta. In discharge 107611, the correction coil is turned off when $\beta_{\mathrm{N}}$ is just above the predicted no-wall limit. Here the drag on the plasma rotation is amplified by the rotationally stabilized, resonant RWM (as discussed later). When the rotation drops below a critical value, the RWM becomes unstable and grows causing a beta collapse. Such macroscopic comparisons between experiment and modeling give another very clear demonstration of the accuracy of the ideal MHD calculations of the nowall stability limit. [12].

Idea-Wall Beta Limit and Disruption Precursor. The MHD instability that ends discharge 106535 at $\beta_{\mathrm{N}}$ twice the no-wall limit is consistent with an $n=1$ kink mode occurring at the stability limit calculated with an ideal wall. About $100 \mathrm{~ms}$ before the disruption, the plasma rotation begins to decrease gradually [Fig. 1(e)], suggesting increased drag due to a marginally stable RWM. Just before the disruption, data from the toroidal array of Mirnov loops [Figs. 3(a-c)] show an $n=1$ structure with a rotation frequency $\omega / 2 \pi=1 \mathrm{kHz}$ and a growth time of $\tau_{\mathrm{g}} \sim 0.3 \mathrm{~ms}$. The relatively rapid rotation and growth $\left(\omega \tau_{\mathrm{w}} \gg 1\right.$ and $\left.\tau_{\mathrm{g}} / \tau_{\mathrm{w}}<<1\right)$ suggest that this instability would behave about the same in the presence of a perfectly conducting wall. The measured growth time can be explained by a model $[13,14]$ for an ideal mode slowly pushing through the stability boundary: $\tau_{\mathrm{g}}=\tau_{\mathrm{MHD}}^{2 / 3} \tau_{\mathrm{h}}^{1 / 3}$, where $\tau_{\mathrm{MHD}}$ is the expected growth time of the ideal MHD mode and $\tau_{h}$ is the time scale for the increase of the instability drive. Here the measured rise time for the instability drive, $\beta_{\mathrm{N}} / \ell_{\mathrm{i}}$, is $\tau_{\mathrm{h}} \sim 2 \mathrm{~s}$, and the measured growth time of the kink mode is $\tau_{\mathrm{g}}=300 \mu \mathrm{s}$, which yield $\tau_{\mathrm{MHD}} \sim 4 \mu \mathrm{s}$. This time is consistent with expectations for ideal MHD time scales. The ideal MHD calculations of the $n=1$ growth rate in presence of an ideal wall were carried out for the equilibrium reconstructed at $\mathrm{t}=$ $2000 \mathrm{~ms}$. The plots of the normalized growth rate versus the plasma-wall separation in Fig. 3(d) show that $\simeq 100 \mathrm{~ms}$ before the disruption the equilibrium is close to marginal stability with the ideal wall at the position of the vacuum vessel. With a slightly higher beta, comparable to the increase in $\beta_{\mathrm{N}} / \ell_{\mathrm{i}}$ at the disruption, the equilibrium is at the instability threshold with respect to wall position and therefore also with respect to beta. These experiments therefore provide a quantitative benchmarking of ideal MHD calculations of both the no-wall and the ideal-wall beta limit. Above all, the results demonstrate that the RWM can be stabilized by rotation at beta values across the entire range $\beta_{\mathrm{N}}^{\text {no wall }} \leq \beta_{\mathrm{N}}<\beta_{\mathrm{N}}^{\text {ideal wall }}$. 

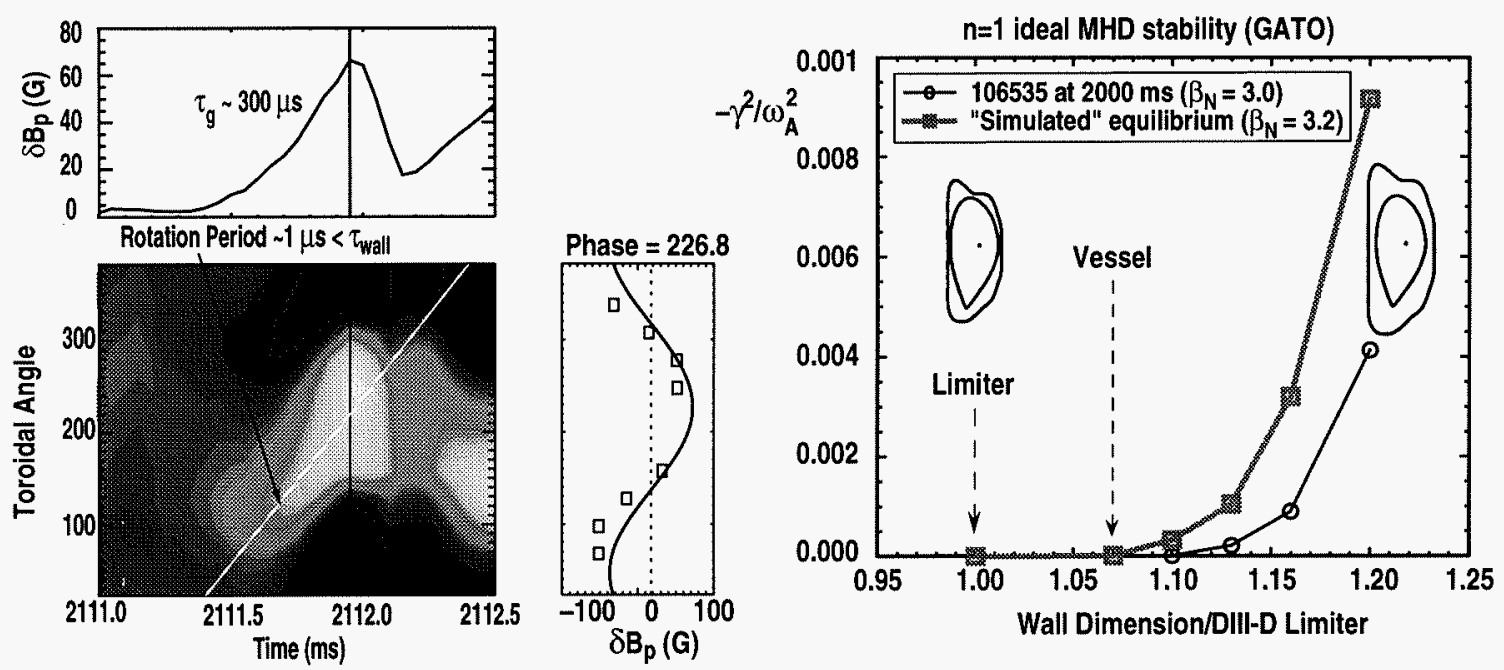

Fig. 3. Magnetic measurements of the fast growing $n=1$ MHD instability in discharge 106535 at $\beta_{N} \approx 2 \beta_{N}^{\text {no wall }}$. (a) Time trace of the $n=1$ amplitude obtained from an array of Mirnov probes distributed around the torus at the outer midplane; (b) contour plot of all the Mirnov probe signals vs. time and toroidal angle; (c) same signals vs. toroidal angle at time of peak amplitude; (d) calculated growth rate of the $n=1$ kink mode vs. assumed position of a perfectly conducting wall for two cases: experimental equilibrium reconstructed at $\sim 100 \mathrm{~ms}$ before the disruption (black) and equilibrium reconstruction obtained after having scaled up by $7 \%$ the pressure profile (gray). The vacuum vessel is well approximated by 1.07 times the limiter surface position.

Re-Tuning of the Error Correction. It was suggested by Boozer [15], that the presence of a slowly growing RWM could be explained as the plasma response to magnetic field asymmetries resonant with the rotationally stabilized RWM. In a real tokamak, $n=1$ asymmetries arise from inevitable small irregularities in positioning coils (poloidal field coils) and current feeds (toroidal field coil). Uncorrected, these asymmetries exert drag on the plasma rotation at the singular surfaces (equivalent to "slipping" in an induction motor model $[16,17])$. Moreover, they can resonate with the RWM, since this is a nearly stationary mode. Experiments [18] designed to test this idea have confirmed that above the $n=1$ ideal $\beta_{\mathrm{N}}$ limit calculated in absence of a conducting wall and plasma rotation, a RWM which is stabilized by a resistive wall and plasma rotation effectively "amplifies" the magnetic field asymmetries ("error field amplification"), increasing the drag on the plasma rotation. As the plasma rotation decreases, even at constant $\beta_{N}$, the RWM becomes closer to its marginal stability boundary, thus the resonant amplification increases. At constant angular momentum injection the process is non-linearly unstable, with a more rapid rotation drop leading to a more rapid increase in the RWM amplitude, until below the rotation threshold for RWM stabilization, a more rapid linear RWM growth $\left(\gamma \sim 1 / \tau_{\mathrm{w}}\right)$ occurs and causes a beta collapse. In some plasmas, the small amplitude RWM (not yet linearly unstable) may cause enough of an energy degradation that $\beta_{\mathrm{N}}$ drops below the no-wall limit, leading to beta saturation, rather than to a large beta collapse. The discovery of error field amplification suggests correction of asymmetries is critical above the no-wall limit and such asymmetries may be responsible for loss of 
RWM stability. Routine $n=1$ correction is done on DIII-D with the C-Coil [7] using an algorithm based both on known and empirical sources of field errors and minimizing a weighted sum of the squares of measured error field amplitudes with poloidal mode numbers $m=1,2$ and 3 . This algorithm and its weights were originally determined from the locked mode onsets of a series of DIII-D discharges at low beta, with monotonic q-profiles having periodic $q=1$ sawteeth instabilities. Re-tuning the algorithm for high beta, $q_{\min } \geq 1.5$ plasmas so as to minimize rotation decay during the high beta phase, indicates that the weighting of the $m / n=2 / 1$ field asymmetry is dominant, and that the previous algorithm finds a similar optimal $n=1$ correction field phase but tends to underestimate the optimal $n=1$ correction amplitude. Figure 4 summarizes the shot-to-shot scan of the amplitude and phase of the $n=1$ correction field, which led to re-tuning the algorithm for high beta, $q_{\min } \geq 1.5$ plasmas. Higher correction currents lead to slower rotation decay [Fig. 4(a,b)], and longer wall stabilized periods with fixed NBI power. Note that the rotation threshold at the $q=2$ surface is independent from the residual asymmetry amplitude. The slowest rotation decay (discharge 103158) is given by correction coil currents of amplitude approximately twice the original correction algorithm (103154). Fitting the plasma rotation data and the residual, uncorrected asymmetry field $\mathbf{B}_{\mathrm{REF}}$ (as the correction field is varied shot-to-shot) with an induction motor model based on a single $\mathrm{m} / \mathrm{n}=$ 2/1 mode, $d f_{\mathrm{p}} / d t=a_{0}+\left(a_{1} / f_{\mathrm{p}}\right) B_{\mathrm{REF}}^{2}$ [Fig. 4(c)], shows a deep minimum in the sum of squared residuals, [Fig. 4(d)] when the amplitude of the intrinsic field asymmetry is increased by $37 \%$, and the phase is shifted by 15 degrees with respect to the originally assumed values, based on low beta, sawteething, $q_{\mathrm{min}}=q_{0} \approx 1$ discharges.

Dynamic Field Symmetrization vs. Improved Correction Algorithm. A new method to attain a similar improved magnetic field symmetry, consists in the use of the RWM feedback system [19-21] to detect and oppose the error field amplification effect when $\beta_{\mathrm{N}}$ is increased above the no-wall limit. Amplification of the field asymmetries by a marginally stable plasma is potentially a very sensitive means of detecting the asymmetries and controlling correction coils. In Fig. 5 the correction currents in the three pairs of opposing C-coils are compared for the two discharges of Fig. 1. Discharge 107603 used the re-tuned correction algorithm, while in discharge 106536 the symmetrization is obtained through use of the RWM feedback (dynamic symmetrization). A high value of the proportional gain is essential to drive sufficient current to correct the asymmetries. Stability of the feedback system at high gain is allowed by using sensors which are highly de-coupled from the driver coils. In this case the sensors measure the poloidal field at the outer midplane, while the driver coils apply an almost purely radial field at the midplane. Because some sensor-driver coupling still exists, the system oscillates at high gain, especially near the maximum current output ( $4.5 \mathrm{kA}$ in this discharge) where the system may not behave linearly. Furthermore, clipping of the oscillations occurs at the maximum 

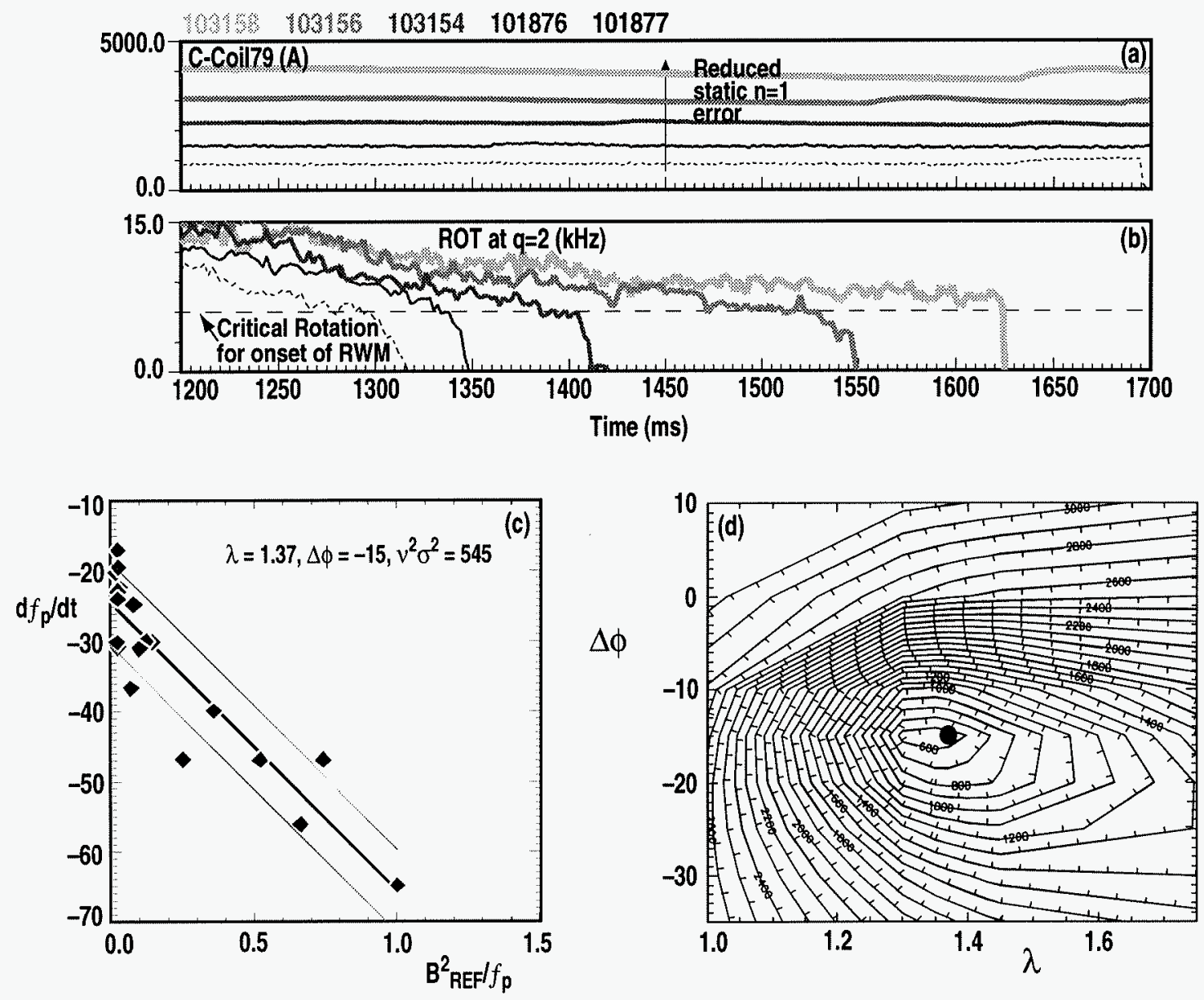

Fig. 4. Increasing magnetic field correction current (a) yields slower plasma rotation decay near the $q=2$ surface (b) in discharges above the no-wall beta limit. According to a simple induction motor model the plasma rotation decay rates measured in discharges with varying amplitude and phase of the $2 / 1$ component of the correction field should be in a linear relationship with $\mathbf{B}_{\mathrm{REF}}^{2} / f_{\mathrm{p}}$, where $\mathbf{B}_{\mathrm{REF}}$ is the residual $2 / 1$ field asymmetry after correction. The amplitude and phase of the assumed intrinsic $2 / 1$ field asymmetry are varied with respect to the originally assumed values through an amplitude multiplier $\lambda$ and a toroidal phase shift $\Delta \phi$, (c) shows the linear fit to the data $( \pm 1 \sigma)$ obtained for the set of $\lambda$ and $\Delta \phi$ corresponding to a deep minimum in (d) the contour plot of $v^{2} \sigma^{2}$ (=sum of squares of residuals) vs. $\lambda$ and $\Delta \phi$. (Here $\sigma$ is the standard error of estimate and $v=N-2$ is the number of degrees of freedom after fitting $N$ discharges with 2 parameters.)

current, which also alters the feedback performance. Overall, the correction current amplitudes are quite similar for the two discharges. The plasma performance is the same until the waveforms for $I_{p}$ and $\beta_{N}$ are programmed to diverge, as shown in Fig. 1 . Potentially, the feedback method avoids the need for explicit measurements of the field asymmetries, or for re-tuning of the correction currents any time the plasma configuration is changed. 
These recent DIII-D experiments demonstrate that passive stabilization of the RWM by resistive wall and plasma rotation is possible, even at $\beta_{\mathrm{N}}$ values significantly above the no-wall limit. Accurate correction of magnetic field asymmetries is critical to minimize plasma rotation decay at beta above the no-wall limit. RWM feedback operation at high stable gain gives a new method to determine the improved magnetic field correction by sensing and opposing the resonant response of the stable RWM to the uncorrected field asymmetry. With improved magnetic field symmetry, and increased angular momentum injection, a path to sustained stable operation, at plasma pressure up to twice the ideal MHD $n=1$ free-boundary pressure limit, has been discovered in the DIII-D tokamak. At twice the free-boundary pressure limit, an MHD instability is observed, which is consistent with having reached the "ideal wall" pressure limit predicted by stability calculations. High plasma performance in the wall stabilized regime appears limited in duration only by the increase in the edge density. The implications of this work are potentially quite profound for the development of steady-state advanced tokamaks, and may allow these devices to operate stably well above the conventional pressure limit for as long as sufficient torque is provided.

This research is sponsored by the U.S. Department of Energy under Contract Nos. DE-AC03-99ER54463, DE-FG02-89ER53297, DE-AC05-00OR22725, and DE-AC02$76 \mathrm{CH} 03073$.

\section{References}

[1] F. Troyon, R. Gruber, H. Saurenmann, S. Semenzato, and S. Succi, Plasma Phys. and Contr. Fusion 26, 209 (1984).

[2] J.P. Freidberg, J.P., Ideal Magnetohydrodynamics, Plenum Press, New York (1987).

[3] A. Bondeson and D.J. Ward, Phys. Rev. Lett. 72, 2709 (1994).

[4] R. Betti and J.P. Freidberg, Phys. Rev. Lett. 74, 2949 (1995).

[5] E.J. Strait, T.S. Taylor, A.D. Turnbull, J.R. Ferron, L.L. Lao, B.W. Rice, O. Sauter, S.J. Thomson, and D. Wròblewski, Phys. Rev. Lett. 74, 2483 (1994). 
[6] A.M. Garofalo, et al., Phys. Rev. Lett. 82, 3811 (1999).

[7] R.J. Buttery., et al., Nucl. Fusion 39, 1827 (1999); J.T. Scoville and R.J. La Haye, "Multi-Mode Error Field Correction in the DIII-D Tokamak," to be submitted to Nucl. Fusion.

[8] D.R. Ernst, B. Coppi, S.D. Scott, M. Porkolab, and TFTR Group, Phys. Rev. Lett. 81, 2454 (1998).

[9] G.T.A. Huysmans, et al., Nucl. Fusion 39, 1489 (1999); A. Isayama, et al., Nucl. Fusion 41, 761 (2001)

[10] L.C. Bernard, F.J. Helton, and R.W. Moore, Comput. Phys. Commun. 21, 377 (1981).

[11] T.S. Taylor, E.J. Strait, L.L. Lao, M.E. Mauel, A.D. Turnbull, K.H. Burrell, M.S. Chu, J.R. Ferron, and R.J. Groebner, Phys. Plasmas 2, 2890 (1995).

[11] R. Fitzpatrick, Phys. Plasmas 5, 3325 (1998).

[12] A.D. Turnbull, et al., "Predictive Capability of MHD Stability Limits in High Performance DIII-D Discharges," to be published in Nucl.Fusion (2001).

[13] J.D. Callen, C.C. Hegna, B.W. Rice, E.H. Strait and A.D. Turnbull, Phys. Plasmas 6, 2963 (1999).

[14] G.L. Jahns, M.S. Chance, et al., Nucl.Fusion 28, 881 (1988).

[15] A.H. Boozer, Phys. Rev. Lett. 86, 1176 (2001).

[16] T.H. Jensen A.W. Leonard, and A.W. Hyatt, Phys. Fluids B 5, 1239 (1993).

[17] R. Fitzpatrick, Phys. Plasmas 5, 3325 (1998).

[18] A.M. Garofalo., et al., Nucl. Fusion 41, 1171 (2001).

[19] A.M. Garofalo, et al., Nucl. Fusion 40, 1491 (2000).

[20] M. Okabayashi, et al., Phys. Plasmas 8, 2071 (2001).

[21] L.C. Johnson et al., in "Structure and Feedback Stabilization of Resistive Wall Modes in DIII-D," in Proceedings of the $28^{\text {th }}$ EPS Conference on Controlled Fusion and Plasma Physics, Madeira, 2001, (European Physical Society, PetitLancy, 2001). 